\title{
An Electrochemical Avenue to Blue Luminescent Nanocrystals from Multiwalled Carbon Nanotubes (MWCNTs)
}

\author{
Jigang Zhou, ${ }^{\dagger}$ Christina Booker,${ }^{\dagger}$ Ruying Li ${ }^{\ddagger}$ Xingtai Zhou, ${ }^{\dagger}$ Tsun-Kong Sham ${ }^{*},{ }^{\dagger}$ \\ Xueliang Sun, ${ }^{*}, \neq$ and Zhifeng Ding ${ }^{*}, \dagger$ \\ Department of Chemistry and Department of Mechanical and Materials Engineering, \\ The University of Western Ontario, London, ON N6A 5B7 Canada
}

Received September 25, 2006; E-mail: zfding@uwo.ca

Luminescent nanostructures are very attractive because of their promised applications in optoelectronic devices, ${ }^{1,2}$ biology labeling, ${ }^{3}$ and biomedicine. ${ }^{4}$ To maintain a benign environment, silicon ${ }^{5-7}$ and carbon ${ }^{8}$ nanostructures are preferred in many fields. The search for a good carbon emitter is very challenging because neither of the two bulk carbon allotropes, graphite and diamond, give strong luminescence, given that the former is a conductor and the latter is an insulator having an indirect band gap of $5.5 \mathrm{eV}$. Molecular and nanostructured carbon forms, including fullerene, single-walled carbon nanotubes (SWCNTs), and multiwalled carbon nanotubes (MWCNTs), show a wealth of properties that are different from their bulk counterparts. ${ }^{9}$ However, these carbon complexes are still not efficient emitters, particularly in the visible region. These carbon nanostructures are being studied in the areas of separation, purification, dispersion, and functionalization. ${ }^{10}$ Some carbon nanostructures have been reported to emit efficiently in the visible range, ${ }^{3,8,11}$ which shows promise in the development of efficient carbon emitters. Here we report a novel method for preparing carbon nanocrystals (NCs), which give off strong blue luminescence, by electrochemical treatment of MWCNTs.

The electrochemical preparation of carbon NCs was performed in a degassed acetonitrile solution with $0.1 \mathrm{M}$ tetrabutylammonium perchlorate (TBAP) as the supporting electrolyte. MWCNTs used in the experiments were grown on a carbon paper by the chemical vapor deposition (CVD) method as described elsewhere. ${ }^{12}$ The MWCNT-covered carbon paper was cut to a suitable size and was placed in a Teflon jacket designed for making an electrical contact and exposing a disk shaped surface. The electrochemical cell consisted of this working electrode, a Pt wire counter electrode, and an $\mathrm{Ag} / \mathrm{AgClO}_{4}$ reference electrode. The applied potential was cycled between -2.0 and $2.0 \mathrm{~V}$ at a scan rate of $0.5 \mathrm{~V} / \mathrm{s}$. Figure 1 shows the cyclic voltammograms (CVs) recorded on a carbon paper (curve a), the paper fully covered with MWCNTs (curve b), and 1000 cycles (curve c). A large capacitor charging current was expected, as is shown by the curves b and $\mathrm{c}$ in Figure 1, given the large surface area. The CV of the CNTs after 1000 cycles (curve c) shows a larger charging current than that after 100 cycles, which in turn, exhibits a larger current than that of pristine CNTs. This can be attributed to the increase of effective electrochemical area. CVs of the MWCNTs demonstrate that oxidations occurred at 0.40 , 0.51 , and $1.7 \mathrm{~V}$ and reductions occurred at -0.71 and $-1.68 \mathrm{~V}$. These peaks can be assigned to redox reactions involving defects and sidewalls. Electrochemical reactions of MWCNTs at a glassy carbon electrode in aqueous solution were reported although the redox peaks were not assigned..$^{13}$ Similar to surface functionalities of porous carbon materials, ${ }^{14}$ these reactions are associated with

\footnotetext{
Department of Chemistry.
}

$¥$ Department of Mechanical and Materials Engineering.

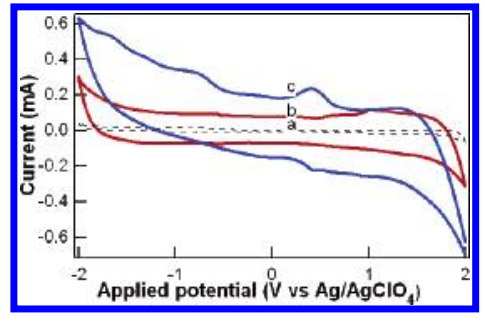

Figure 1. Cyclic voltammetry of carbon paper (a), pristine CNTs (b), and treated CNTs (c) in acetonitrile with $0.1 \mathrm{M}$ TBAP supporting electrolyte.

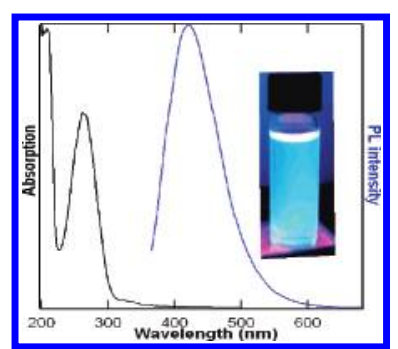

Figure 2. UV-vis absorption and PL spectra of carbon NCs in acqueous solution. PL spectrum was obtained under excitation at $365 \mathrm{~nm}$. Inset is the solution illuminated by an UV lamp.

surface oxygen complexes and increasing defect densities in MWCNTs, which increase the hydrophilicity.

The electrolyte solution was found to change from colorless to yellow and finally to a dark brown color that emitted blue luminescence upon irradiation with an UV lamp. The luminescent, water soluble product was purified by evaporating the acetonitrile from the solution, dissolving the remaining solid in water, and then dialyzing the aqueous solution with a cellulose ester membrane bag. These processes removed most supporting electrolyte, TBAP, from the NCs. The UV-vis absorption spectrum shown in Figure 2 reveals that the first absorption band at $270 \mathrm{~nm}(4.59 \mathrm{eV})$ has a narrow fwhm of $50 \mathrm{~nm}(0.86 \mathrm{eV})$. The photoluminescence (PL) spectrum shows a peak position at $410 \mathrm{~nm}(3.02 \mathrm{eV})$, a $140 \mathrm{~nm}$ $(1.57 \mathrm{eV})$ red shift from the absorption first peak (Figure 2). The inset in Figure 2 shows bright blue emission from the product excited by the UV lamp as we monitored the solution during the electrochemical process. The emission spectrum also shows the excitation dependent feature (Figure S1 in the Supporting Information (SI)). The quantum yield was determined to be 0.064 , excited at $340 \mathrm{~nm}$ (Figure S2 in SI).

Surprisingly, carbon NCs were observed by examining the product with high-resolution electron transmission microscopy (Figure 3a). It can be seen clearly that high-density carbon NCs have a uniform spherical shape and a narrow size distribution being $2.8 \pm 0.5 \mathrm{~nm}$ in diameter. These results agree with the distribution information drawn from the absorption and PL spectroscopy shown in Figure 2. ${ }^{15}$ NCs likely have variable emission sites and/or surface 


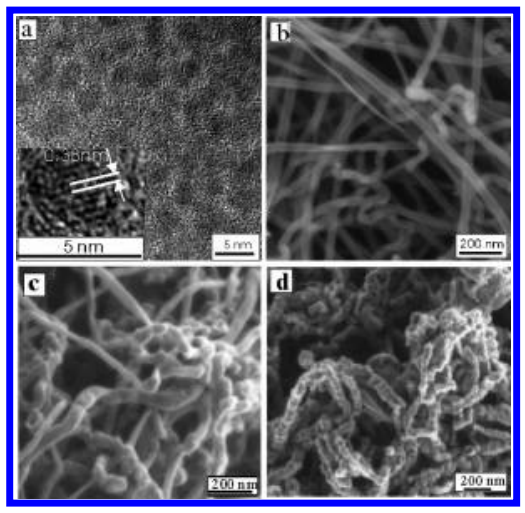

Figure 3. HRTEM images of carbon NCs (a); SEM images of pristine MWCNTs (b); MWCNTs after 100 cycles where the applied potential was scanned between 2.0 and $-2.0 \mathrm{~V}$ at $0.5 \mathrm{~V} / \mathrm{s}$ (c); and MWCNTs after 1000 cycles (d). Inset in panel a is the HRTEM image of a typical carbon NC.

states on individual NCs. ${ }^{8}$ Raman spectrum (Figure S6 in SI) shows the characteristics of both $\mathrm{sp}^{2}$ and disordered carbons. Furthermore, the observed lattice spacing distance is from 3.1 to $3.4 \AA$ (Figure S3 in SI). The inset in Figure 3a illustrates a lattice spacing of $3.3 \AA$ that is very close to the graphite 002 lattice spacing. ${ }^{9}$ The spacing is confirmed by XRD (Figure S7 in SI). This lattice contraction in the above range is common for materials in nano scale regime.

Since our MWCNTs were formed with scrolled graphene layers (SI), we envision that TBA cations most probably intercalated into the gaps during electrochemical cycling and broke the tubes near the defects. Carbon NCs were then exfoliated, entering into the electrolyte solutions. While electrochemical intercalation of some organic molecules into CNTs was reported in the literature, ${ }^{16}$ this is the first time that the breaking of CNTs to generate luminescent carbon NCs by cycling the applied potential is observed.

The structure evolution of the MWCNTs during electrochemical treatments was monitored by SEM ex situ as shown in Figure 3bd. Before the electrochemical cycling, the as-synthesized MWCNTs reveal very straight and well isolated shapes (Figure 3b). However, after the treatments, the MWCNTs become entangled together with swelling and curled features (Figure 3c). Further treatment results in serious deformation, and openings along the tube walls (Figure $3 \mathrm{~d})$. These results are consistent with the $\mathrm{CV}$ results, showing an increase in active area and surface reactions during the cycling. It is clear that the electrochemical treatment process is very complex in this system, and the details are still under investigation in our lab.

The carbon paper as well as the substrate deposited with $\mathrm{Co} / \mathrm{Ni}$ catalyst by $\mathrm{CVD}^{12}$ were treated following the same electrochemical procedure as described above. No NCs were obtained. This confirms that the luminescent carbon NCs were generated from the CNTs rather than the carbon paper with the catalyst. When the same procedure was applied to the CNTs in the potential window of an aqueous solution with either $\mathrm{KCl}$, or $\mathrm{KClO}_{4}$ as the electrolyte, no luminescence was observed. This indicates that TBA plays a role in the electrochemical process.
The luminescence mechanism of this carbon emitting material is still not clearly understood..$^{3,8,11}$ Besides $\mathrm{C}_{60}$ adducts ${ }^{11 \mathrm{a}, \mathrm{f}}$ and carbon nanotubes in aromatic amine solvent ${ }^{11 \mathrm{~b}}$ from which the luminescence is from the charge transfer from $\mathrm{N}$ to carbon, a widely accepted mechanism for luminescent NCs is the radiative recombination of excitons from not only carbon NCs of different sizes in the sample but also a distribution of different emissive trap sites (Figure S1 in SI). ${ }^{8}$ This is similar to luminescence from silicon NCs. ${ }^{5,6}$

In conclusion, we have developed a method by employing electrochemistry to convert MWNTs into highly efficient luminescent carbon NCs, which can be easily dispersed in various solvents. It is anticipated that these NCs will find a wealth of applications in biology labeling and optoelectronic devices.

Acknowledgment. Financial support from NSERC, OPC, CFI, OIT, PREA, and UWO is acknowledged.

Supporting Information Available: Complete ref 8, additional PL spectra, quantum yield calculations, TEM and Raman, and XRD spectra. This material is available free of charge via the Internet at http:// pubs.acs.org.

\section{References}

(1) Bard, A. J.; Ding, Z.; Myung, N. In Structure and Bonding; Springer: Berlin, 2005; Vol. 118, pp 1-57.

(2) Hutchison, K.; Gao, J.; Schick, G.; Rubin, Y.; Wudl, F. J. Am. Chem. Soc. 1999, 121, 5611-5612.

(3) Lin, Y.; Zhou, B.; Martin, R. B.; Henbest, K. B.; Harruff B. A.; Riggs J. E.; Guo, Z.-X.; Allard L. F.; Sun, Y.-P. J. Phvs. Chem. B 2005, 109, 14779-14782.

(4) Michalet, X.; Pinaud, F. F.; Bentolila, L. A.; Tsay, J. M.; Doose, S.; Li, J. J.; Sundaresan, G.; Wu, A. M.; Gambhir, S. S.; Weiss, S. Science 2005 $307,538-544$

(5) Wilson, W. L.; Szajowski, P. F.; Brus, L. E. Science 1993, 262, 12421244.

(6) Ding, Z.; Quinn, B. M.; Haram, S. K.; Pell, L. E.; Korgel, B. A.; Bard, A. J. Science 2002, 296, 1293-1297.

(7) Sham, T. K.; Jiang, D. T.; Coulthard, I.; Lorimer, J. W.; Feng, X. H.; Tan, K. H.; Frigo, S. P.; Rosenberg, R. A.; Houghton, D. C.; Bryskiewicz B. Nature 1993, 363, 331-334.

(8) Sun, Y.-P.; et al. J. Am. Chem. Soc. 2006, 128, 7756-7757.

(9) Iijima, S. Nature 1991, 354, 56-58.

(10) Huang, W.; Lin, Y.; Taylor, S.; Gaillard, J.; Rao, A. M.; Sun, Y.-P. Nano Lett. 2002, 2, 231-234.

(11) (a) Cheng, J. X.; Fang, Y.; Huang, Q. J.; Yan, Y. J.; Li, X. Y. Chem. Phys. Lett. 2000, 330, 262-266. (b) Riggs, J. E.; Guo, Z.; Carroll, D. L.; Sun, Y.P. $J$. Am. Chem. Soc. 2000, 122, 5879-5880. (c) Schick, G.; Levitus, M.; Kvetko, L.; Johnson, B. A.; Lamparth, I.; Lunkwitz, R.; Ma B.; Khan, S. I.; Garcia-Garibay, M. A.; Rubin, Y. J. Am. Chem. Soc. 1999, 121, 3246-3247. (d) Sun, Y.; Wilson, S. R.; Schuster, D. I. J. Am. Chem. Soc. 2001, 123, 5348-5349. (e) Xu, X.; Ray, R.; Gu, Y.; Ploehn, H. J.; Gearheart, L.; Raker, K.; Scrivens, W. A. J. Am. Chem. Soc. 2004 126, 12736-12737. (f) Zhang, F.; Fang, Y. J.Phys. Chem. B 2006, 110 9022-9026.

(12) Sun, X.; Stansfield, B.; Dodelet, J. P.; Desilets, S. Chem. Phys. Lett. 2002 $363,415-421$

(13) Li, Y.; Shi, X.; Hao, J. Carbon 2006, 44, 2661-2670.

(14) Bleda-Martinez, M. J.; Lozano-Castello, D.; Morallon, E.; Cazorla-Amoro, D.; Linares-Solano, A. Carbon 2006, 44, 2642-2651.

(15) Murray, C. B.; Norris, D. J.; Bawendi, M. G. J. Am. Chem. Soc. 1993 $115,8706-8715$.

(16) Wildgoose, G. G.; Hyde, M. E.; Lawrence, N. S.; Leventis, H. C.; Jiang, L.; Jones, T. G. J.; Compton, R. G. Langmuir 2005, 21, 4584-4591.

JA0669070 